\title{
E-optimal Designs for Three Treatments
}

\author{
Valentin Parvu and J. P. Morgan \\ Virginia Tech
}

$25^{\text {th }}$ March 2005

\begin{abstract}
E-optimality is studied for three treatments in an arbitrary $n$-way heterogeneity setting. In some cases maximal trace designs cannot be E-optimal. When there is more than one E-optimal design for a given setting, the best with respect to all reasonable criteria is determined. Key words and phrases: design optimality, multi-way heterogeneity, row-column design
\end{abstract}

\section{Introduction}

Of the many settings with multiple blocking factors, by far the most common are those where the arrangement of experimental units can be taken as an $n$-dimensional hyperrectangle for some $n \geq 2$. If $b_{j}$ is the number of levels of the $j$ th blocking factor, then $b_{1} \times b_{2} \times \cdots \times b_{n}$ is the size of the hypperrectangle, and there is no loss of generality in taking $b_{1} \leq b_{2} \leq \cdots \leq b_{n}$. Each cell corresponds to a single experimental unit, to be assigned one of $v$ treatments. Denoting the total number of experimental units by $m=b_{1} b_{2} \ldots b_{n}$, the size of each block of factor $j$, that is, the block size in direction $j$, is $m b_{j}^{-1}$. The standard linear model is:

$$
y=\mu 1+A_{d} \tau+\sum_{j=1}^{n} L_{j} \beta_{j}+\varepsilon,
$$

where $y$ is the $m \times 1$ vector of yields, 1 is a vector of ones, $L_{j}$ is the $m \times b_{j}$ plot-block incidence matrix in direction $j$ of the hyperrectangle with corresponding $b_{j} \times 1$ block effects vector $\beta_{j}$, and $\varepsilon$ is a vector of uncorrelated random errors with zero means and equal variances. The design problem 
is to optimize comparisons of members of the $v \times 1$ treatment effects vector $\tau$, and the precision with which these comparisons are made is controlled by the assignment pattern of treatments to units, that is, by the choice of the $m \times v$ design matrix $A_{d}$.

Morgan and Bailey (2000) recently took a broad look at optimal allocations for multiple blocking factors, allowing for various combinations of nesting, crossing, and other relationships in the block factors structure. Readers are referred there for citations to past work as well as examples of such designs in practice. Like virtually all available theory, Morgan and Bailey's (2000) optimality results for the hyperrectangle described above place restrictions as a function of $v$ on the collection of numbers $\left(b_{1}, \ldots, b_{n}\right)$ that can be quite severe. The same is seen, of course, in any design text: if you wish to compare 5 treatments, say, with two crossed blocking factors, neither texts nor the general literature will offer you much advice unless at least one of $b_{1}$ and $b_{2}$ is a multiple of 5 . A consequence is that many experiments get "forced" into a standard design category, changing $v$ and/or the $b_{i}$ 's according to the limited options available. Statistical theory does not serve science well in these instances.

This paper takes a step towards resolving this situation, and in doing so helps illuminate the considerable technical difficulties involved. The E-optimality question is attacked, and solved, for $v=3$ treatments and any $\left(b_{1}, \ldots, b_{n}\right)$. Sonnemann (1985) has previously solved the optimality question for $v=2$ in two-way heterogeneity settings, and those results are easily extended to $n$-way. Here the extension is not simple.

\section{Information matrix and other preliminaries}

Comparisons of competing designs are made through the $v \times v$ information matrix, also referred to as the $C$-matrix. Computation of the $C$-matrix for hyperrectangle block structure is simple using Morgan and Bailey's (2000) projection method. A mechanical derivation and explicit expression can be found in Cheng (1978), who deals specifically with this structure. The notation adopted here is also taken from Cheng (1978).

Let $N_{j}=\left(n_{i j l}\right)$ be the $v \times b_{j}$ incidence matrix between the $v$ treatments and the $b_{j}$ levels of factor 
$j$. By Theorem 2.1 in Cheng (1978), the $C$-matrix for design $d$ is

$$
C_{d}=D_{r}-\frac{1}{m} \sum_{j=1}^{n} b_{j} N_{j} N_{j}^{\prime}+\frac{n-1}{m} D_{r} 11^{\prime} D_{r}^{\prime},
$$

where $D_{r}=\operatorname{diag}\left(r_{1}, \ldots, r_{v}\right)$ is the diagonal matrix of replication numbers for the treatments. It follows that the $i$ th diagonal element of $C_{d}$ is

$$
c_{i}=r_{i}-\frac{1}{m} \sum_{j=1}^{n}\left(b_{j} \sum_{l=1}^{b_{j}} n_{i j l}^{2}\right)+\frac{n-1}{m} r_{i}^{2}
$$

where $n_{i j l}$ is the number of times treatment $i$ occurs in block $l$ of factor $j$. Each of $r_{i}$ and $n_{i j l}$, and of course $c_{i}$, is a function of the design choice $d$ through $A_{d}$; this is taken to be understood, so that the notational complexity can be eased by not subscripting these quantities with $d$. A useful convention is to label the treatments so that the $r_{i}$ 's are in nonincreasing order: $r_{1} \geq r_{2} \geq \ldots$. This ordering will be maintained throughout this paper.

Any $C$-matrix is symmetric, non-negative definite, and has row and column sums of zero. Consequently, the $C$-matrix for a design with three treatments can be written solely in terms of its diagonal elements:

$$
C_{d}=\left(\begin{array}{ccc}
c_{1} & \frac{1}{2}\left(-c_{1}-c_{2}+c_{3}\right) & \frac{1}{2}\left(-c_{1}+c_{2}-c_{3}\right) \\
\frac{1}{2}\left(-c_{1}-c_{2}+c_{3}\right) & c_{2} & \frac{1}{2}\left(c_{1}-c_{2}-c_{3}\right) \\
\frac{1}{2}\left(-c_{1}+c_{2}-c_{3}\right) & \frac{1}{2}\left(c_{1}-c_{2}-c_{3}\right) & c_{3}
\end{array}\right),
$$

where $c_{1}, c_{2}$, and $c_{3}$ correspond to treatments 1,2 , and 3 , and are given in (3). The two nonzero eigenvalues of the matrix given in (4) are $\frac{1}{2}\left[\sum c_{i} \pm \sqrt{2 \sum_{i<j}\left(c_{i}-c_{j}\right)^{2}}\right]$. E-optimal designs maximize the quantity $Z_{d}$, the smaller of the two eigenvalues:

$$
Z_{d}=\frac{1}{2}\left[\sum c_{i}-\sqrt{2 \sum_{i<j}\left(c_{i}-c_{j}\right)^{2}}\right] .
$$

The expression for $Z_{d}$ simplifies when some of the $c_{i}$ 's are equal. These will be used often:

$$
\begin{aligned}
& c_{1}=c_{2} \geq c_{3} \Rightarrow Z_{d}=\frac{3}{2} c_{3} \\
& c_{1} \geq c_{2}=c_{3} \Rightarrow Z_{d}=c_{2}+c_{3}-\frac{1}{2} c_{1}
\end{aligned}
$$

In some cases there are multiple designs which are E-optimal. With three treatments, the weak majorization order can always discriminate among them. 
Definition 2.1. A design for three treatments is said to be E-M-optimal if (i) it is E-optimal, and (ii) it maximizes the largest eigenvalue of $C_{d}$ amongst all E-optimal designs.

An E-M-optimal design is, for instance, A-best of all E-optimal designs. Indeed, it is best (amongst all E-optimal designs) with respect to every criterion expressed as the sum of a decreasing function of the eigenvalues. The "M" is chosen in accord with the usage by Bagchi and Bagchi (2001), as weak majorization is implied.

Next stated are two useful, well-known bounds for the smallest eigenvalue of a $C$-matrix. These bounds can be derived by the averaging technique described by Constantine (1981), or by other methods as given by Jacroux (1980).

Lemma 2.1. For a design d with information matrix $C_{d}=\left(c_{i i^{\prime}}\right)$, the minimum non-zero eigenvalue $Z_{d}$ satisfies

$$
Z_{d} \leq \frac{v}{v-1} \min _{i}\left(c_{i i}\right)
$$

Lemma 2.2. For a design d with information matrix $C_{d}=\left(c_{i i^{\prime}}\right)$, the minimum non-zero eigenvalue $Z_{d}$ satisfies

$$
Z_{d} \leq \min _{i \neq i^{\prime}} \frac{c_{i i}+c_{i^{\prime} i^{\prime}}-2 c_{i i^{\prime}}}{2}
$$

One more concept is integral to the optimality arguments to follow.

Definition 2.2. In a design with multiple blocking factors, the assignment of treatment $i$ is said to be uniform in direction $j$, if $\left|n_{i j l}-n_{i j l^{\prime}}\right| \leq 1$ for all $l$ and $l^{\prime}$. The assignment of treatment $i$ is uniform if it is uniform in all directions. The design is said to be uniform if all treatments are assigned uniformly in all directions.

"Treatment $i$ is uniform" will be shorthand for "the assignment of treatment $i$ is uniform." If treatment $i$ is uniform in direction $j$, then $\sum_{l} n_{i j l}^{2}$ is minimized for a given replication $r_{i}$, and can be written in terms of $r_{i}$ and $b_{j}$ as:

$$
h\left(r_{i}, b_{j}\right)=r_{i}+\left(2 r_{i}-b_{j}\right) \operatorname{int}\left(\frac{r_{i}}{b_{j}}\right)-b_{j}\left[\operatorname{int}\left(\frac{r_{i}}{b_{j}}\right)\right]^{2} .
$$

In general, if treatment $i$ is uniform in the entire design, then (3) becomes:

$$
c_{i}=r_{i}-\frac{1}{m} \sum_{j=1}^{n}\left(b_{j} h\left(r_{i}, b_{j}\right)\right)+\frac{n-1}{m} r_{i}^{2} .
$$


It is important to realize that neither uniformity of a treatment, nor of the entire design, demands any particular values for the replication numbers $r_{i}$. Obviously, if treatments $i$ and $i^{\prime}$ have the same replication but $i$ is uniform while $i^{\prime}$ is not, then $c_{i^{\prime}}<c_{i}$. For three treatments, the maximin replication is $r=\operatorname{int}\left(\frac{m}{3}\right)$. A design is as close as possible to having equal replication if $r_{1} \leq r_{3}+1$.

Simple manipulation of function $h(r, b)$ defined in (8) gives

$$
\Delta h(r, b)=h(r+1, b)-h(r, b)=1+\frac{2}{b}\left(r-r_{(b)}\right)
$$

where $r_{(b)}=r \bmod b$ (compare Morgan, 1997). For any design with treatment $i$ uniform in direction $j, \sum_{l=1}^{b_{j}} n_{i j l}^{2}=h\left(r_{i}, b_{j}\right)$. The nonuniformity of treatment $i$ in direction $j$ is

$$
N U_{i(j)}=\frac{b_{j}}{m}\left[\sum_{l=1}^{b_{j}} n_{i j l}^{2}-h\left(r_{i}, b_{j}\right)\right]
$$

The total nonuniformity of treatment $i$ is $N U_{i}=\sum_{j=1}^{n} N U_{i(j)}$, which is zero if $i$ is uniform.

Suppose that the number of experimental units is $m \equiv 0 \bmod 3$; this only occurs if at least one of the $b_{j}$ is a multiple of three. For this setting, $r=\frac{m}{3}$ and one can easily construct a uniform design $d^{0}$ with $r_{1}=r_{2}=r_{3}=r$. For this design the information matrix $C_{d^{0}}$ is completely symmetric, and $Z_{d^{0}}=\frac{3 c_{i}^{0}}{2}$. Any design $d$ with either $r_{3}<r$, or with nonuniformity in some treatment, will have $C_{d}$ with at least one diagonal element $c_{i}<c_{i}^{0}$. Then by lemma $2.1, Z_{d}<\frac{3 c_{i}^{0}}{2}=Z_{d^{0}}$, and $d$ is E-inferior to $d^{0}$. In fact, $d^{0}$ is a Youden hyperrectangle, or YHR, which is universally optimal (see Corollary 3.1.2 in Cheng (1978)).

Open are the cases where equal replication is not possible, that is, the cases for which no $b_{j}$ is a multiple of 3. Section 3 lays the groundwork for the general problem by determining E-optimal designs for an unstructured set of blocks $(n=1)$. Sections 4 and 5 solve the settings for $n \geq 2$ for $m \equiv 1 \bmod 3$ and $m \equiv 2 \bmod 3$, respectively. Concluding remarks are in section 6.

\section{One blocking factor}

In the one-way heterogeneity setting $\mathcal{D}(3, b, k)$, with three treatments to be compared in $b$ blocks of $k$ experimental units each, the total number of units is $m=b k$. Let treatment $i$ have replication $r_{i}$, 
with block-wise replications $n_{i l}, l=1,2, \ldots, b$. For such a design $d$, the diagonal elements of $C_{d}$ are $c_{i}=r_{i}-\frac{1}{k} \sum_{l=1}^{b}\left(n_{i l}\right)^{2}$. If treatment $i$ is uniform, then $c_{i}^{0}=r_{i}-\frac{1}{k} h\left(r_{i}, b\right)$, so that the nonuniformity of treatment $i$ is $N U_{i} \equiv N U_{i(1)}=c_{i}^{0}-c_{i} \geq 0$ as given by (11).

\subsection{Block designs with $b k \equiv 1 \bmod 3$}

In this case the maximin replication is $r=\frac{b k-1}{3}$. Consider a uniform design $d^{0}$, with replications $r_{1}=r+1$ and $r_{2}=r_{3}=r$. By (7) the E-value for this design is

$$
Z_{d^{0}}=2 c_{2}^{0}-\frac{c_{1}^{0}}{2}
$$

It will be first shown that $d^{0}$ is E-superior to any design not as close as possible to equal replication.

Lemma 3.1. Block designs with $r_{3} \leq r-1$ cannot be E-optimal for bk $\equiv 1 \bmod 3$.

Proof. It will be shown that $Z_{d^{0}}-\frac{3}{2} c_{3}>0$, where $c_{3}$ is the diagonal element of a uniform treatment with replication $r_{3}=r-1$. This implies $Z_{d^{0}}-\frac{3}{2} c_{3}>0$ for any $r_{3} \leq r-1$, and thus by Lemma 2.1 the result.

Computing the values of the diagonal elements of $C_{d^{0}}$ gives

$$
\begin{gathered}
c_{1}^{0}=r+1-\frac{1}{k} h(r+1, b)= \begin{cases}\frac{2}{9 k}\left(b k^{2}-b+k-1\right), & \text { when } b \equiv 1 \text { and } k \equiv 1 ; \\
\frac{2}{9 k}\left(b k^{2}-b+k+1\right), & \text { when } b \equiv 2 \text { and } k \equiv 2 ;\end{cases} \\
c_{2}^{0}=c_{3}^{0}=r-\frac{1}{k} h(r, b)= \begin{cases}\frac{1}{9 k}\left(2 b k^{2}-2 b-k+1\right), & \text { when } b \equiv 1 \text { and } k \equiv 1 \\
\frac{1}{9 k}\left(2 b k^{2}-2 b-k-1\right), & \text { when } b \equiv 2 \text { and } k \equiv 2 .\end{cases}
\end{gathered}
$$

Suppose a design $d$ has treatment 3 uniform with $r_{3}=r-1$. Then by (9):

$$
c_{3}= \begin{cases}\frac{2}{9 k}\left(b k^{2}-b-2 k+2\right), & \text { when } b \equiv 1 \text { and } k \equiv 1 ; \\ \frac{2}{9 k}\left(b k^{2}-b-2 k-2\right), & \text { when } b \equiv 2 \text { and } k \equiv 2,\end{cases}
$$

from which

$$
Z_{d^{0}}-\frac{3}{2} c_{3}=2 c_{2}^{0}-\frac{c_{1}^{0}}{2}-\frac{3}{2} c_{3}= \begin{cases}\frac{k-1}{3 k}, & \text { when } b \equiv 1 \text { and } k \equiv 1 \\ \frac{k+1}{3 k}, & \text { when } b \equiv 2 \text { and } k \equiv 2,\end{cases}
$$

and the proof is done. 
So all the E-optimal designs must have the same replication numbers as $d^{0}$. Now uniformity of the treatment assignment will be investigated. It will be seen that while treatments 2 and 3 must be uniform, E-optimality can demand that treatment 1 not be uniform. Before embarking on the proof, some relationships will be derived for designs uniform in treatments 2 and 3 .

To begin, suppose $b \equiv k \equiv 1 \bmod 3$. With treatments 2 and 3 uniform, their block-wise replications are $n_{2 l}, n_{3 l} \in\left\{\operatorname{int}\left(\frac{r}{b}\right), \operatorname{int}\left(\frac{r}{b}\right)+1\right\}$, where $\operatorname{int}\left(\frac{r}{b}\right)=\frac{b k-1}{3 k}=\frac{k-1}{3}$. Treatment 1 will be nonuniform in block $l$ if and only if $n_{2 l}=n_{3 l}=\frac{k+2}{3}$ which would make $n_{1 l}=\frac{k-4}{3}$. Thus (see (11)) the nonuniformity $N U_{1}$ of treatment 1 is $\frac{2}{k} x$, where $x$ is the number of blocks in which $n_{1 l}=\frac{k-4}{3}$.

Similarly, when $b \equiv k \equiv 2 \bmod 3$ and treatments 2 and 3 are uniform, the nonuniformity $N U_{1}$ of treatment 1 is $\frac{2}{k} x$, where $x$ is the number of blocks in which $n_{1 l}=\frac{k+4}{3}$.

Then for any design uniform in treatments 2 and $3, c_{1}=c_{1}^{0}-N U_{1}$. Establishing E-optimality will require knowledge of the maximum nonuniformity of treatment 1, that is, the largest possible value of $N U_{1}$, given that treatments 2 and 3 are constrained to be uniform. This maximum is obtained with the following block assignments:

$$
\begin{array}{cccccccc}
\multicolumn{4}{c}{b \equiv 1 \text { and } k \equiv 1} & \multicolumn{3}{c}{b \equiv 2 \text { and } k \equiv 2} \\
n_{1 j} & n_{2 j} & n_{3 j} & \text { no. of blocks } & n_{1 j} & n_{2 j} & n_{3 j} & \text { no. of blocks } \\
\frac{k+2}{3} & \frac{k-1}{3} & \frac{k-1}{3} & \frac{2 b+1}{3} & \frac{k-2}{3} & \frac{k+1}{3} & \frac{k+1}{3} & \frac{2 b-1}{3} \\
\frac{k-4}{3} & \frac{k+2}{3} & \frac{k+2}{3} & \frac{b-1}{3}=x \max & \frac{k+4}{3} & \frac{k-2}{3} & \frac{k-2}{3} & \frac{b+1}{3}=\text { xmax }
\end{array}
$$

where $x \max$ denotes the maximum number of blocks in which treatment 1 is nonuniform.

Let $D$ denote the difference between the diagonal elements of $d^{0}$ :

$$
D=c_{1}^{0}-c_{2}^{0}=\left\{\begin{array}{ll}
\frac{k-1}{3 k}, & \text { when } b \equiv k \equiv 1 ; \\
\frac{k+1}{3 k}, & \text { when } b \equiv k \equiv 2
\end{array} .\right.
$$

The idea is to maintain uniformity in treatments 2 and 3 , but to make treatment 1 nonuniform in such a way that $c_{1}$ is as close as possible to $c_{2}^{0}$. Consider a design $d^{*}$ uniform in treatments 2 and 3 and with treatment 1 nonuniform in $x^{*}=\min \left[x \max , \operatorname{int}\left(\frac{D}{2 / k}\right)\right]$ blocks. Note that

$$
\operatorname{int}\left(\frac{D}{2 / k}\right)= \begin{cases}\operatorname{int}\left(\frac{k-1}{6}\right), & \text { when } b \equiv k \equiv 1 ; \\ \operatorname{int}\left(\frac{k+1}{6}\right), & \text { when } b \equiv k \equiv 2 .\end{cases}
$$


Theorem 3.1. In $\mathcal{D}(3, b, k)$ with $b k \equiv 1 \bmod 3$, design $d^{*}$ is E-M-optimal.

Proof. By (7), the E-value of $d^{*}$ is

$$
Z_{d^{*}}=2 c_{2}^{0}-\frac{1}{2} c_{1}^{*}=\frac{3}{2} c_{2}^{0}-\frac{1}{2}\left(D-\frac{2}{k} x^{*}\right) .
$$

First, consider settings where $x \max \geq \operatorname{int}\left(\frac{D}{2 / k}\right)$ and so $x^{*}=\operatorname{int}\left(\frac{D}{2 / k}\right)$. Designs which are nonuniform in treatments 2 and 3 will be eliminated first, followed by designs which are nonuniform in treatment 1 in a different number of blocks than $d^{*}$.

By $(16), D-\frac{2}{k} x^{*} \leq \frac{1}{k}$, and so

$$
Z_{d^{*}} \geq \frac{3}{2} c_{2}^{0}-\frac{1}{2 k}
$$

Any design $d$ nonuniform in treatment 2 will have $c_{2} \leq c_{2}^{0}-\frac{2}{k}$, and by Lemma $2.1, Z_{d} \leq \frac{3}{2} c_{2}<Z_{d^{*}}$. Therefore, any design nonuniform in treatment 2 will be E-inferior to $d^{*}$. By symmetry, the same result holds for designs nonuniform in treatment 3.

Any design $d$ uniform in treatments 2 and 3 and with treatment 1 nonuniform in less than $x^{*}$ blocks will have $c_{1}>c_{1}^{*}$. So by $(7)$ and (19), $Z_{d}=2 c_{2}^{0}-\frac{c_{1}}{2}<Z_{d^{*}}$. Any design $d$ uniform in treatments 2 and 3 with treatment 1 nonuniform in more than $x^{*}=\operatorname{int}\left(\frac{D}{2 / k}\right)$ blocks will have $c_{1} \leq c_{2}^{0}-\frac{1}{k}$ (see (16)). Then (6) and (19) $\Rightarrow Z_{d}=\frac{3}{2} c_{3}<Z_{d^{*}}$.

Now consider settings where $x \max <\operatorname{int}\left(\frac{D}{2 / k}\right)$, so $x^{*}=x \max$. Using (12), (13), and (14),

$$
Z_{d^{*}}=2 c_{2}^{0}-\frac{1}{2}\left(c_{1}^{0}-x \max \frac{2}{k}\right)=Z_{d^{0}}+\frac{x \max }{k}=\frac{b k-1}{3} .
$$

By (4) and Lemma 2.2, it is known that for any $d$, an upper bound for $Z_{d}$ is $u b_{d}=c_{2}+c_{3}-\frac{c_{1}}{2}$. As usual, let $n_{i l}$ denote the number of times treatment $i$ appears in block $l$. Now write $n_{1 l}=n_{1}+e_{l}$, where $n_{1}=\operatorname{int}\left(\frac{r_{1}}{b}\right)=\operatorname{int}\left(\frac{b k+2}{3 b}\right)$, and $\sum_{l=1}^{b} e_{l}=r_{1}-b n_{1}=r+1-b n_{1}$. The $e_{l}$ 's are the deviations from equal block-wise replications for treatment 1 . For a given set of $e_{l}$ 's, $u b_{d}$ is maximized when $c_{2}+c_{3}=2 r-\frac{1}{k} \sum_{l=1}^{b}\left(n_{2 l}^{2}+n_{3 l}^{2}\right)$ is maximized. Thus the assignment pattern for the $n_{2 l}$ 's and $n_{3 l}$ 's that maximizes $c_{2}+c_{3}$ for given assignment of treatment 1 (and thus $c_{1}$ ) is

$$
n_{2 l}=n_{3 l}=\frac{k-n_{1 l}}{2}=\frac{1}{2}\left(k-n_{1}-e_{l}\right) .
$$

Note that when $x^{*}=x \max , d^{*}$ is a special case of this assignment pattern (as shown by the blockwise replications (15) for $d^{*}$ ). In general, call this assignment pattern $\bar{d}$. Although a design with 
these block-wise replications does not exist if $k-n_{1}-e_{l}$ is odd for some $l$, the bound $u b_{\bar{d}} \equiv \overline{u b}$ will be useful in showing the optimality of $d^{*} . C_{\bar{d}}$ has diagonal elements $c_{1}=r+1-\frac{1}{k} \sum_{l=1}^{b}\left(n_{1}+e_{l}\right)^{2}$ and $c_{2}=c_{3}=r-\frac{1}{4 k} \sum_{l=1}^{b}\left(k-n_{1}-e_{l}\right)^{2}$ so that

$$
\begin{aligned}
\overline{u b} & =2 c_{2}-\frac{c_{1}}{2}=\frac{3}{2} r-\frac{1}{2}-\frac{1}{2 k} \sum_{j=1}^{b}\left[\left(k-n_{1}-e_{j}\right)^{2}-\left(n_{1}+e_{j}\right)^{2}\right] \\
& =\frac{3}{2} r-\frac{1}{2}-\frac{1}{2} \sum_{j=1}^{b}\left(k-2 n_{1}-2 e_{j}\right)=\frac{3}{2} r-\frac{1}{2}-\frac{1}{2}[b k-2(r+1)] \\
& =r=\frac{b k-1}{3} .
\end{aligned}
$$

A key observation is that the upper limit $\overline{u b}$ does not depend on the values of the $e_{l}$ 's!

Now $Z_{d^{*}}=\overline{u b}$, which means that $d^{*}$ is E-optimal. The only question remaining is whether there are other E-optimal designs. Again, $Z_{d} \leq u b_{d} \leq \overline{u b}$, and $u b_{d}$ does not attain $\overline{u b}$ for most designs. For a given assignment of treatment 1 , if $n_{2 l} \neq n_{3 l}$ for some $l$, then $u b<\overline{u b}$. But $n_{2 l}=n_{3 l}$ for all $l \Rightarrow c_{2}=c_{3}$. If $c_{1}<c_{2}=c_{3}$, then (6) gives $Z_{d}=\frac{3 c_{1}}{2}<\overline{u b}$. Therefore, for $x \max <\operatorname{int}\left(\frac{\operatorname{diff}}{2 / k}\right)$, a design can be E-optimal only if $n_{2 l}=n_{3 l}$ for all $l$, and $c_{1} \geq c_{2}$.

If $x \max <\operatorname{int}\left(\frac{\operatorname{diff}}{2 / k}\right)$ and other E-optimal designs exist, $d^{*}$ is E-M-optimal because it has higher trace, and $C_{d}$ has only two non-zero eigenvalues.

Example 3.1. As a simple example of a design, other than $d^{*}$, which is E-optimal, consider two blocks of size 50. Here are the block assignments for designs $d^{*}$ and $d^{\prime}$, both E-optimal with minimum eigenvalue $Z_{d^{*}}=Z_{d^{\prime}}=r=33$. These designs are E-superior to the uniform design $d^{0}$ which places treatment 1 on 17 units in each block.

$d^{*}:$\begin{tabular}{|c|ccc|}
\hline$j$ & $n_{1 j}$ & $n_{2 j}$ & $n_{3 j}$ \\
\hline 1 & 16 & 17 & 17 \\
2 & 18 & 16 & 16 \\
\hline
\end{tabular}

$d^{\prime}:$\begin{tabular}{|c|ccc|}
\hline$j$ & $n_{1 j}$ & $n_{2 j}$ & $n_{3 j}$ \\
\hline 1 & 14 & 18 & 18 \\
2 & 20 & 15 & 15 \\
\hline
\end{tabular}




\subsection{Block designs with $b k \equiv 2 \bmod 3$}

Theorem 3.2. In $\mathcal{D}(3, b, k)$ with $b k \equiv 2 \bmod 3$, the E-M-optimal designs are uniform with replications $r_{1}=r_{2}=\frac{b k+1}{3}$, and $r_{3}=\frac{b k-2}{3}$.

Proof. Here the maximin replication is $r=\frac{b k-2}{3}$. Consider a uniform design $d^{0}$ with $r_{1}=r_{2}=r+1$, and $r_{3}=r$. Then $c_{1}^{0}=c_{2}^{0}>c_{3}^{0}$, and the E-value for $d^{0}$ is $Z_{d^{0}}=\frac{3}{2} c_{3}^{0}$ by (6).

Any design $d$ with $r_{3} \leq r$ will have $c_{3} \leq c_{3}^{0}$, and therefore $Z_{d} \leq Z_{d^{0}}$, by Lemma 2.1. By the same argument, any other design with $r_{i}=r$ and not uniform in treatment $i$ is E-inferior to $d^{0}$. Therefore $d^{0}$ is E-optimal.

Since $d^{0}$ is uniform, $C_{d^{0}}$ has maximum trace amongst all block designs. Since $C_{d}$ has only two non-zero eigenvalues, $d^{0}$ is E-M-optimal. Any nonuniform design will have smaller trace, so no nonuniform design can be E-M-optimal.

Uniform designs with $r_{i}<r$ are E-inferior to $d^{0}$ by lemma 2.1, leaving only one possible competitor. A uniform design $d$ with $r_{1}=r+2$, and $r_{2}=r_{3}=r$ has $c_{1}>c_{3}^{0}$ and $c_{2}=c_{3}=c_{3}^{0}$. For these values (7) gives $Z_{d}=2 c_{3}^{0}-\frac{1}{2} c_{1}<Z_{d^{0}}$.

\section{Experiment size $m \equiv 1 \bmod 3$}

For this setting the maximin replication is $r=\frac{m-1}{3}$. Consider a uniform design $d^{0}$ with $r_{1}=r+1$ and $r_{2}=r_{3}=r$, call it $d^{0}$. By (7) the E-value for this design

$$
Z_{d^{0}}=2 c_{2}^{0}-\frac{c_{1}^{0}}{2}
$$

Similar to lemma 3.1, it will be shown that $d^{0}$ is E-superior to any design with $r_{3} \leq r-1$, reducing the class of E-competitors.

Lemma 4.1. Designs for which $r_{3} \leq r-1$ cannot be E-optimal for $m \equiv 1 \bmod 3$ if $n>2$, or if $n=2$ and $\left(b_{1}, b_{2}\right) \neq(4,4)$.

Proof. It will be shown that $Z_{d^{0}}-\frac{3}{2} c_{3} \geq 0$, where $c_{3}$ is the diagonal element of a uniform treatment 
with replication $r_{3}=r-1$ and $Z_{d^{0}}$ is given by (20). This implies that $Z_{d^{0}}-\frac{3}{2} c_{3} \geq 0$ for any $r_{3} \leq r-1$, and thus by Lemma $2.1, d^{0}$ is E-superior to any design which has $r_{3} \leq r-1$.

To do this, the following identities are required:

$$
\begin{gathered}
r \bmod b_{j}=r_{\left(b_{j}\right)}= \begin{cases}\frac{b_{j}-1}{3} & \text { when } b_{j} \equiv 1 \bmod 3 ; \\
\frac{2 b_{j}-1}{3} & \text { when } b_{j} \equiv 2 \bmod 3 .\end{cases} \\
r-1 \quad \bmod b_{j}=(r-1)_{\left(b_{j}\right)}= \begin{cases}\frac{b_{j}-4}{3} & \text { when } b_{j} \equiv 1 \bmod 3 ; \\
\frac{2 b_{j}-4}{3} & \text { when } b_{j} \equiv 2 \bmod 3 .\end{cases}
\end{gathered}
$$

Note that $(r-1)_{\left(b_{j}\right)}=r_{\left(b_{j}\right)}-1$, so

$$
\begin{aligned}
b_{j}\left[2 h\left(r, b_{j}\right)\right. & \left.-\frac{1}{2} h\left(r+1, b_{j}\right)-\frac{3}{2} h\left(r-1, b_{j}\right)\right]=-b_{j}\left[\frac{1}{2} \Delta h\left(r, b_{j}\right)-\frac{3}{2} \Delta h\left(r-1, b_{j}\right)\right] \\
& =-b_{j}\left[\frac{1}{2}+\frac{1}{b_{j}}\left(r-r_{\left(b_{j}\right)}\right)-\frac{3}{2}-\frac{1}{b_{j}}\left(r-1-(r-1)_{\left(b_{j}\right)}\right)\right] \\
& =b_{j}+2\left(r-r_{\left(b_{j}\right)}\right)
\end{aligned}
$$

Now using (20) and (9):

$$
\begin{aligned}
Z_{d^{0}}-\frac{3}{2} c_{3}= & 2 r-\frac{r+1}{2}-\frac{3(r-1)}{2}+\frac{n-1}{m}\left[2 r^{2}-\frac{1}{2}(r+1)^{2}-\frac{3}{2}(r-1)^{2}\right] \\
& -\frac{1}{m} \sum_{j=1}^{n} b_{j}\left[2 h\left(r, b_{j}\right)-\frac{1}{2} h\left(r+1, b_{j}\right)-\frac{3}{2} h\left(r-1, b_{j}\right)\right] \\
\stackrel{(23)}{=} & 1+\frac{2}{m}(n-1)(r-1)-\frac{1}{m} \sum_{j=1}^{n}\left(b_{j}+2 r-2-2(r-1)_{\left(b_{j}\right)}\right) \\
= & \frac{1}{m}\left(m+2(n-1)(r-1)-\sum b_{j}-2 n r+2 n+2 \sum(r-1)_{\left(b_{j}\right)}\right) \\
= & \frac{1}{m}\left(m-2 r+2-\sum b_{j}+2 \sum(r-1)_{\left(b_{j}\right)}\right) \\
= & \frac{1}{3 m}\left(m+8-3 \sum b_{j}+6 \sum(r-1)_{\left(b_{j}\right)}\right),
\end{aligned}
$$

since $r=\frac{m-1}{3}$. Write $y=\prod b_{j}+8-3 \sum b_{j}+6 \sum(r-1)_{\left(b_{j}\right)} ; y \geq 0$ will be shown by induction. For the first induction step, check inequality for $n=2$, with the two cases:

1. $b_{1} \equiv b_{2} \equiv 1 \bmod 3 \Rightarrow y \stackrel{(22)}{=}\left(b_{1}-1\right)\left(b_{2}-1\right)-9 \geq 0$ with equality if and only if $b_{1}=b_{2}=4$.

2. $b_{1} \equiv b_{2} \equiv 2 \bmod 3 \Rightarrow y \stackrel{(22)}{=}\left(b_{1}+1\right)\left(b_{2}+1\right)-9 \geq 0$ with equality if and only if $b_{1}=b_{2}=2$ (in which case no connected design exists). 
By $(22),(r-1)_{\left(b_{j}\right)} \geq \frac{b_{j}-4}{3}$, with equality when $b_{j} \equiv 1 \bmod 3$, so $y \geq \prod b_{j}-\sum b_{j}-8(n-1)$.

For the second induction step, assume for $n \geq 3$ that $y \geq 0$ for $n-1$ factors, that is, $\prod_{j=1}^{n-1} b_{j} \geq$ $\sum_{j=1}^{n-1} b_{j}+8(n-2)$. This implies that $\prod_{j=1}^{n} b_{j} \geq\left[\sum_{j=1}^{n-1} b_{j}+8(n-2)\right] b_{n}>\sum_{j=1}^{n} b_{j}+8(n-1)$. Therefore $y \geq 0$ for any $n \geq 2$, and $y=0$ if and only if $n=2$ and $b_{1}=b_{2}=4$ or $b_{1}=b_{2}=2$.

Lemma 4.1 leaves open the possibility of different replications for the $4 \times 4$ layout. This case is easily disposed of by complete enumeration, which shows there are two E-optimal designs:

$d_{1}:$\begin{tabular}{|c|c|c|c|}
\hline 1 & 2 & 3 & 1 \\
\hline 2 & 3 & 1 & 2 \\
\hline 3 & 1 & 2 & 3 \\
\hline 1 & 2 & 3 & 1 \\
\hline
\end{tabular}

Both designs are uniform, with replication vectors $(6,5,5)$ and $(6,6,4)$, respectively.

Henceforth assume $r_{1}=r+1, r_{2}=r_{3}=r$. Now uniformity of the treatment assignment will be investigated. It will be seen that while treatments 2 and 3 must be uniform, E-optimality can demand that treatment 1 not be uniform. Before embarking on the proof, some relationships will be derived for the case of treatments 2 and 3 uniform.

To begin, suppose $b_{j} \equiv 1 \bmod 3$, which means that $m b_{j}^{-1} \equiv 1 \bmod 3$. Every block of factor $j$ has $m b_{j}^{-1}$ cells. With treatments 2 and 3 uniform, their block-wise replications are $n_{2 j l}, n_{3 j l} \in$ $\left\{\operatorname{int}\left(\frac{r}{b_{j}}\right), \operatorname{int}\left(\frac{r}{b_{j}}\right)+1\right\}$, and by $(21) \operatorname{int}\left(\frac{r}{b_{j}}\right)=\frac{m b_{j}^{-1}-1}{3}$. Treatment 1 will be nonuniform in block $l$ of factor $j$ if and only if $n_{2 j l}=n_{3 j l}=\frac{m b_{j}^{-1}+2}{3}$ which would make $n_{1 j l}=\frac{m b_{j}^{-1}-4}{3}$. Thus (see (11)) the nonuniformity $N U_{1(j)}$ of treatment 1 due to factor $j$ is $\frac{2}{m b_{j}^{-1}} x_{j}$, where $x_{j}$ is the number of blocks of factor $j$ in which $n_{1 j l}=\frac{m b_{j}^{-1}-4}{3}$. Then for any design uniform in treatments 2 and $3, c_{1}=c_{1}^{0}-N U_{1}$.

Establishing E-optimality will require knowledge of the maximum nonuniformity of treatment 1 in factor $j$, that is, the largest possible value of $N U_{1(j)}$, given that treatments 2 and 3 are constrained to be uniform. This maximum is obtained with the following block assignments:

$$
\begin{array}{cccc}
\multicolumn{5}{c}{b_{j} \equiv 1} & \bmod 3 \\
n_{1 j l} & n_{2 j l} & n_{3 j l} & \text { no. of blocks } \\
\frac{m b_{j}^{-1}+2}{3} & \frac{m b_{j}^{-1}-1}{3} & \frac{m b_{j}^{-1}-1}{3} & \frac{2 b_{j}+1}{3} \\
\frac{m b_{j}^{-1}-4}{3} & \frac{m b_{j}^{-1}+2}{3} & \frac{m b_{j}^{-1}+2}{3} & \frac{b_{j}-1}{3}=x \max _{j}
\end{array}
$$


where $\operatorname{xmax}_{j}$ is the maximum number of blocks in which treatment 1 can be nonuniform in factor $j$. Similarly, when $b_{j} \equiv 2 \bmod 3, N U_{1(j)}=\frac{2}{m b_{j}^{-1}} x_{j}$, where $x_{j}$ is the number of blocks of factor $j$ in which $n_{1 j l}=\frac{m b_{j}^{-1}-4}{3}$. This is obtained with these block assignments:

$$
\begin{array}{cccc}
\multicolumn{5}{c}{b_{j} \equiv 2 \bmod 3} \\
n_{1 j l} & n_{2 j l} & n_{3 j l} & \text { no. of blocks } \\
\frac{m b_{j}^{-1}-2}{3} & \frac{m b_{j}^{-1}+1}{3} & \frac{m b_{j}^{-1}+1}{3} & \frac{2 b_{j}-1}{3} \\
\frac{m b_{j}^{-1}+4}{3} & \frac{m b_{j}^{-1}-2}{3} & \frac{m b_{j}^{-1}-2}{3} & \frac{b_{j}+1}{3}=x \max _{j}
\end{array}
$$

where now $\operatorname{xmax}_{j}=\frac{b_{j}+1}{3}$.

Design $d^{0}$ was defined above as the uniform design with replications $r_{1}=r+1$, and $r_{2}=r_{3}=r$. Let $D$ denote the difference between $c_{1}^{0}$ and $c_{2}^{0}$.

$$
\begin{aligned}
D & =c_{1}^{0}-c_{2}^{0}=1-\frac{1}{m} \sum_{j=1}^{n} b_{j} \Delta h\left(r, b_{j}\right)+\frac{n-1}{m}(2 r+1)= \\
& =\frac{2 m n+m+n-1}{3 m}-\frac{1}{m} \sum_{j=1}^{n}\left[b_{j}+2\left(r-r_{\left(b_{j}\right)}\right)\right]
\end{aligned}
$$

Now competitors to $d^{0}$ can be defined. The idea is to maintain uniformity in treatments 2 and 3 , but to make treatment 1 nonuniform in such a way that $c_{1}$ is as close as possible to $c_{2}^{0}$. Thus consider two designs, call them $d^{*}$ and $d_{*}$, where for $d^{*}, c_{1}^{*} \geq c_{2}^{0}$, and for $d_{*}, c_{1 *} \leq c_{2}^{0}$. To find the number of blocks $x_{j}^{*}$ and $x_{j *}$ of factor $j$ in which treatment 1 should be made nonuniform, solve the following integer minimization/maximization problems:

$$
\begin{aligned}
& \operatorname{maximize} \frac{2}{m} \sum_{j=1}^{n}\left(b_{j} x_{j}^{*}\right), \quad \text { subject to } 0 \leq x_{j}^{*} \leq x \max _{j} \text { and } \frac{2}{m} \sum_{j=1}^{n}\left(b_{j} x_{j}^{*}\right) \leq D \\
& \text { minimize } \frac{2}{m} \sum_{j=1}^{n}\left(b_{j} x_{j *}\right), \quad \text { subject to } 0 \leq x_{j *} \leq x \max _{j} \text { and } \frac{2}{m} \sum_{j=1}^{n}\left(b_{j} x_{j *}\right) \geq D
\end{aligned}
$$

Note that (29) may not have a solution. This occurs exactly when $\frac{2}{m} \sum_{j=1}^{n}\left(b_{j} x_{\max }\right)<D$.

Theorem 4.1. For $m \equiv 1 \bmod 3$, if $n>2$, or if $n=2$ and $\left(b_{1}, b_{2}\right) \neq(4,4)$, E-M-optimal designs have the same block assignments as either $d^{*}$ or $d_{*}$. Furthermore, $d^{*}$ is E-M-optimal if and only if $\frac{1}{m} \sum_{j=1}^{n} b_{j}\left(3 x_{j *}-x_{j}^{*}\right) \leq D$ or $\frac{2}{m} \sum_{j=1}^{n}\left(b_{j} x \max _{j}\right)<D$. 
When $n=2$ and $b_{1}=b_{2}=4$, an E-M-optimal design different from $d^{*}$ and $d_{*}$ exists, as discussed following lemma 4.1.

Proof. The E-values for the two proposed designs are

$$
Z^{*}=2 c_{2}^{0}-\frac{c_{1}^{*}}{2} \quad \text { and } \quad Z_{*}=\frac{3}{2} c_{1 *}
$$

where $c_{1}^{*}=c_{2}^{0}+D-\frac{2}{m} \sum_{j=1}^{n}\left(b_{j} x_{j}^{*}\right)$, and $c_{1 *}=c_{2}^{0}+D-\frac{2}{m} \sum_{j=1}^{n}\left(b_{j} x_{j *}\right)$. It can be easily seen that $Z^{*} \geq Z_{*}$ if and only if $D \geq \frac{1}{m} \sum_{j=1}^{n} b_{j}\left(3 x_{j *}-x_{j}^{*}\right)$. In case of equality, design $d^{*}$ is E-M better because $c_{1}^{*}>c_{1 *}$.

By lemmas 2.1 and 2.2, the E-value of any design $d$ with $c_{2}=c_{3}=c_{2}^{0}$ is $Z_{d}=\left\{\begin{array}{ll}2 c_{2}^{0}-\frac{c_{1}}{2} & \text { if } c_{1} \geq c_{2}^{0} \\ \frac{3}{2} c_{1} & \text { if } c_{1}<c_{2}^{0}\end{array}\right.$. The conditions on $d^{*}$ and $d_{*}$ imply $Z^{*}>2 c_{2}^{0}-\frac{c_{1}}{2}$ if $c_{1} \geq c_{2}^{0}$, and $Z_{*}>\frac{3}{2} c_{1}$ if $c_{1}<c_{2}^{0}$. The goal is to show that any design nonuniform in treatments 2 or 3 cannot have a higher E-value than both $d^{*}$ and $d_{*}$, so that E-M-optimal designs must have $c_{2}=c_{3}=c_{2}^{0}$.

First, note that by the definition of $d^{*}$, if $c_{1}^{*}-c_{2}^{0} \geq \frac{2 b_{j}}{m}$ then $x_{j}^{*}=x \max _{j}$, otherwise treatment 1 could have been made nonuniform in one more block of factor $j$. Also $c_{1}^{*}-c_{2}^{0} \geq \frac{2 b_{j}}{m}$ implies one of the following two statements are true:

1. $x_{j *}=0$, for otherwise making treatment 1 nonuniform in $x_{j *}-1$ blocks in direction $j$ would bring $c_{1}$ closer to $c_{2}^{0}$ than at least one of $c_{1}^{*}$ or $c_{1 *}$;

2. $x_{j}^{*}=\operatorname{xmax}_{j}$ for all $j$ and equation (29) has no solution.

In the latter situation, $d^{*}$ will be shown to be E-M-optimal (see the last paragraph of this proof). Now suppose

$$
\begin{aligned}
& c_{1}^{*}-c_{2}^{0} \geq \frac{2 b_{j}}{m} \text { for all } j<s \\
& c_{1}^{*}-c_{2}^{0}<\frac{2 b_{j}}{m} \text { for all } j \geq s
\end{aligned}
$$

for some $s \leq n$. It follows that

$$
x_{j}^{*}=x \max _{j} \text { and } x_{j *}=0 \text { for all } j<s .
$$


This implies that $Z^{*}=2 c_{2}^{0}-\frac{c_{1}^{*}}{2}>\frac{3}{2} c_{2}^{0}-\frac{b_{j}}{m}$ for all $j \geq s$. Then any design which has treatment 2 nonuniform in any direction $j \geq s$ will have $c_{2} \leq c_{2}^{0}-\frac{2 b_{j}}{m}$, and so by Lemma 2.1, (30) and (31), $Z_{d} \leq \frac{3}{2} c_{2} \leq \frac{3}{2} c_{2}^{0}-\frac{3 b_{j}}{m}<Z^{*}$; this also applies for any design that has treatment 3 nonuniform in any direction $j \geq s$.

Thus, E-optimal designs have treatments 2 and 3 uniform in any direction $j \geq s$. Let $N U_{1(\geq s)}$ denote the nonuniformity of treatment 1 in directions $j \geq s$.

$$
N U_{1(\geq s)}=\sum_{j=s}^{n} N U_{i(j)}=\frac{1}{m} \sum_{j=s}^{n} b_{j}\left[\sum_{l=1}^{b_{j}} n_{1 j l}^{2}-h\left(r+1, b_{j}\right)\right]
$$

Similarly, define $N U_{1(<s)}$ as the nonuniformity of treatment 1 in directions $j<s$. Also let $N U_{1(\geq s)}^{*}$ and $N U_{1(\geq s *)}$ represent treatment 1 nonuniformity in directions $j \geq s$ in designs $d^{*}$ and $d_{*}$. By (28) and (29) there is no design $d$ uniform in treatments 2 and 3 with $c_{1 *}<c_{d 1}<c_{1}^{*}$. Suppose there exists a design $d^{\prime}$ uniform in treatments 2 and 3 in directions $j \geq s$ which has $N U_{1(\geq s)}^{*}<N U_{1(\geq s)}^{\prime}<$ $N U_{1(\geq s *)}$. It is claimed that this is not possible because it would contradict the preceding statement:

1. If $N U_{1(\geq s)}^{\prime} \geq D$, arranging treatments 1,2 and 3 uniformly in directions $j<s$ of $d^{\prime}$, will result in $c_{1 *}<c_{1}^{\prime} \leq c_{1}^{0}-D<c_{1}^{*}$ since $N U_{<s *}=0$ by $(32)$.

2. If $D-\frac{2 b_{s-1}}{m}<N U_{1(\geq s)}^{\prime}<D$, arranging treatments 1,2 and 3 uniformly in directions $j<s$ of $d^{\prime}$, will result in $c_{1 *} \leq c_{1}^{0}-D<c_{1}^{\prime}<c_{1}^{*}$, since by $(31), c_{1}^{*}-c_{2}^{0} \geq \frac{2 b_{j}}{m}$ for all $j<s$

3. If $N U_{1(\geq s)}^{\prime} \leq D-\frac{2 b_{s-1}}{m}$ then $c_{1}^{\prime}-c_{2}^{0} \geq \frac{2 b_{j}}{m}$ for all $j<s$ if all treatments of $d^{\prime}$ are uniform in all directions $j<s$. Note that $c_{1}^{*}-c_{2}^{0} \geq \frac{2 b_{j}}{m}$ for all $j<s$, as well. Now, keeping treatments 2 and 3 uniform in all directions, take $x_{j}^{\prime}=\operatorname{xmax}_{j}=x_{j}^{*}$ for all $j<s$, where treatment 1 of $d^{\prime}$ is nonuniform in $x_{j}^{\prime}$ blocks of direction $j<s$. Then $c_{1}^{\prime}<c_{1}^{0}-D$ by definition of $c_{1}^{*}$ and the fact that $N U_{1(\geq s)}^{*}<N U_{1(\geq s)}^{\prime}$, and so $c_{1}^{\prime}<c_{1 *}$ by definition of $c_{1 *}$. Now decrease $x_{1}^{\prime}$ one unit at a time down to 0 , then decrease $x_{2}^{\prime}$ one unit at a time down to 0 , and so on, stopping as soon as $c_{1}^{\prime}>c_{1}^{0}-D$ is achieved. Due to the ordering on the $b_{j}$ 's and consequently on the step sizes this procedure takes in changing $c_{1}^{\prime}$, and since $N U_{1(\geq s)}^{*}<N U_{1(\geq s)}^{\prime}$, the ending value must satisfy $c_{1}^{\prime}<c_{1}^{*}$.

Each case says $c_{1 *}<c_{1}^{\prime}<c_{1}^{*}$ with a uniform arrangement of treatments 2 and 3 in $d^{\prime}$, which contradicts (28) and (29). Hence, there is no competitor design, uniform in treatments 2 and 
3 in directions $j \geq s$, which has $N U_{1(\geq s)}^{*}<N U_{1(\geq s)}<N U_{1(\geq s *)}$. Also, any design which has $N U_{1(\geq s)}>N U_{1(\geq s *)}$, will have $c_{1}<c_{1^{*}}$ by (32), and thus $Z_{d}<Z_{*}$. Therefore any competitor must have treatments 2 and 3 uniform in directions $j \geq s$, and the nonuniformity of treatment 1 in directions $j \geq s$ must be $N U_{1(\geq s)} \leq N U_{1(\geq s)}^{*}$.

By lemma 2.2, any design $d$ satisfies $Z_{d} \leq c_{2}+c_{3}-\frac{c_{1}}{2}$. Call this upper bound $u b_{d}$. Using (3) and (9), for the competitors remaining, the bound can be computed as:

$$
\begin{aligned}
u b_{d}= & 2 r-\frac{r+1}{2}+\frac{n-1}{m}\left(2 r^{2}-\frac{(r+1)^{2}}{2}\right)-\frac{1}{m} \sum_{j=1}^{n}\left[b_{j} \sum_{l=1}^{b_{j}}\left(n_{2 j l}^{2}+n_{3 j l}^{2}-\frac{n_{1 j l}^{2}}{2}\right)\right] \\
= & \text { const }_{1}-\frac{1}{m} \sum_{j=1}^{s-1}\left[b_{j} \sum_{l=1}^{b_{j}}\left(n_{2 j l}^{2}+n_{3 j l}^{2}-\frac{n_{1 j l}^{2}}{2}\right)\right] \\
& -\frac{1}{m} \sum_{j=s}^{n}\left[2 b_{j} h\left(r, b_{j}\right)-b_{j} \frac{h\left(r+1, b_{j}\right)}{2}\right]+\frac{1}{2} N U_{1(\geq s)} \\
= & \text { const }_{2}-\frac{1}{m} \sum_{j=1}^{s-1}\left[b_{j} \sum_{l=1}^{b_{j}}\left(n_{2 j l}^{2}+n_{3 j l}^{2}-\frac{n_{1 j l}^{2}}{2}\right)\right]+\frac{1}{2} N U_{1(\geq s)}
\end{aligned}
$$

where const $_{1}$ and const $_{2}$ are constants, depending only on $s$ and the dimensions of the hyperrectangle. Expression (34) depends on the nonuniformity of treatment 1 in every direction, and of treatments 2 and 3 in directions $j<s$. Note that $d^{*}$ reaches its bound since $Z^{*}=2 c_{2}^{0}-\frac{c_{1}^{*}}{2}=u b^{*}$.

Next it is shown that $u b_{d}$ for any other design uniform in treatments 2 and 3 in directions $j \geq s$, and with $N U_{1(\geq s)} \leq N U_{1(\geq s)}^{*}$, cannot be higher than $u b^{*}$. Since $N U_{1(\geq s)} \leq N U_{1(\geq s)}^{*}$, it is sufficient to show that $d^{*}$ minimizes the sum $\sum_{l=1}^{b_{j}}\left(n_{2 j l}^{2}+n_{3 j l}^{2}-\frac{n_{1 j l}^{2}}{2}\right)$ for each $j \leq s-1$. Given a set of block assignments for treatment $1,\left(n_{1 j 1}, n_{1 j 2}, \ldots, n_{1 j b_{j}}\right)$, this sum is minimized by setting $n_{2 j l}=n_{3 j l}=$ $\frac{1}{2}\left(m b_{j}^{-1}-n_{1 j l}\right)$ for all $l$, with value

$\sum_{l=1}^{b_{j}}\left(n_{2 j l}^{2}+n_{3 j l}^{2}-\frac{n_{1 j l}^{2}}{2}\right)=\sum_{l=1}^{b_{j}} \frac{1}{2}\left[\left(m b_{j}^{-1}-n_{1 j l}\right)^{2}-n_{1 j l}^{2}\right]=\frac{m^{2}}{2 b_{j}}-m b_{j}^{-1} \sum_{l=1}^{b_{j}} n_{1 j l}=\frac{m^{2}}{2 b_{j}}-m b_{j}^{-1}(r+1)$

This value is the same for any $\left(n_{1 j 1}, n_{1 j 2}, \ldots, n_{1 j b_{j}}\right)$, as long as $n_{2 j l}=n_{3 j l}=\frac{1}{2}\left(m b_{j}^{-1}-n_{1 j l}\right)$ for all $l$. For $d^{*}$ this is achieved (see (32) and the block assignments in direction $j$ when $x_{j}^{*}=x m a x_{j}$ given by $(25)$ and $(26))$. Thus $u b$ is maximized by $d^{*}$.

This also proves that $d^{*}$ is E-optimal when (29) has no solution (i.e. when $x_{j}^{*}=\operatorname{xmax}_{j}$ for all $j$ ). In this case $d^{*}$ reaches the absolute maximum of (34) because $n_{2 j l}^{*}=n_{3 j l}^{*}$ for any $j$ and $l$. 
In some cases, E-optimal designs other than $d^{*}$ or $d_{*}$ might exist, having treatments 2 and 3 nonuniform. However, $C_{d^{*}}$ or $C_{d_{*}}$ will have higher trace, meaning these competitors are inferior with respect to every criterion depending on both eigenvalues.

For a given hyperrectange of size $b_{1} \times b_{2} \times \cdots \times b_{n}$, equations (28) and (29) must be solved numerically. A Mathematica program that computes $x_{j}^{*}$ and $x_{j *}$ for all $j$, and decides whether $d^{*}$ or $d_{*}$ is E-Moptimal, is available from the first author.

For convenience, some E-M-optimal assignments for 2 and 3 blocking factors are given in Table 1 and Table 2, respectively. The 4 -tuples in Table 1 are $\left(b_{1}, b_{2} ; x_{1}, x_{2}\right)$ for a $b_{1} \times b_{2}$ row-column design with $x_{1}$ units of nonuniformity in rows and $x_{2}$ in columns, $b_{1} \leq b_{2} \leq 20$. For example, a $5 \times 8$ E-M-optimal row-column design has treatment 1 nonuniform in 0 rows and 1 column. Here is one such design, with treatment 1 nonuniform in the first column:

\begin{tabular}{|l|l|l|l|l|l|l|l|}
\hline 1 & 2 & 3 & 1 & 2 & 3 & 1 & 2 \\
\hline 3 & 1 & 2 & 3 & 1 & 2 & 3 & 1 \\
\hline 2 & 3 & 1 & 2 & 3 & 1 & 2 & 3 \\
\hline 1 & 2 & 3 & 1 & 2 & 3 & 1 & 2 \\
\hline 1 & 3 & 2 & 3 & 1 & 2 & 3 & 1 \\
\hline
\end{tabular}

Similarly, Table 2 contains 6 -tuples $\left(b_{1}, b_{2}, b_{2} ; x_{1}, x_{2}, x_{3}\right)$ for 3 -dimensional hyperrectangles and $b_{1} \leq$ $b_{2} \leq b_{3} \leq 10$, where $x_{j}$ is the number of blocks of factor $j$ where treatment 1 is nonuniform.

At least for two blocking factors, achievement of the required nonuniformity is usually accomplished quickly by trial and error. The construction problem has been solved in its full generality, for any number $n$ of factors, in Parvu (2004).

\section{$5 \quad$ Experiment size $m \equiv 2 \bmod 3$}

For this setting the maximin replication is $r=\frac{m-2}{3}$. Create a uniform design $d^{0}$ with $r_{1}=r_{2}=r+1$, and $r_{3}=r$. Then (6) says $Z_{d^{0}}=\frac{3}{2} c_{3}^{0}$. Any design $d$ must have some $r_{i} \leq r$, implying $c_{i} \leq c_{3}^{0}$ and hence $Z_{d} \leq \frac{3}{2} c_{i} \leq Z_{d^{0}}$. Therefore $d^{0}$ is E-optimal, and since uniformity implies maximal trace of the information matrix, it is E-M optimal. The main result of this section says that there are no 
other E-M-optimal designs. There are, however, other E-optimal designs.

Theorem 5.1. For $m \equiv 2(\bmod 3)$, E-M-optimal designs are uniform with replications $r_{1}=r_{2}=$ $\frac{m+1}{3}, r_{3}=\frac{m-2}{3}$.

Proof. Competitors with $r_{3}<r$ have $c_{3}<c_{3}^{0}$, and hence by lemma 2.1, $Z_{d}<Z_{d^{0}}$. For $r_{3} \geq r$, the only replication numbers different from those of $d^{0}$ are $r_{1}=r+2, r_{2}=r_{3}=r$. For such a design $d$, if either treatment 2 or treatment 3 is nonuniform, then again lemma 2.1 says $Z_{d}<Z_{d^{0}}$. Thus $d$ must have $c_{2}=c_{3}$ and so $(7) \Rightarrow Z_{d}=c_{2}+c_{3}-\frac{1}{2} c_{1}=2 c_{3}^{0}-\frac{1}{2} c_{1} \leq \frac{3}{2} c_{3}^{0}$ with equality if and only if $c_{1}=c_{2}=c_{3}=c_{3}^{0}$. But if this condition for E-optimality is met, trace of $C_{d}$ is not maximal.

The proof says E-optimal (but not E-M-optimal) designs can be constructed whenever for replications $(r+2, r, r)$ and treatments 2 and 3 both uniform, treatment 1 can be made nonuniform in such a way that $c_{1}=c_{2}=c_{3}$; this is only sometimes possible. E-optimality without trace maximization can also be achieved with replications $(r+1, r+1, r)$, as follows. First, as already shown, treatment 3 must be uniform and so $c_{3}=c_{3}^{0}$. Now for fixed $c_{1}+c_{2}$ and $c_{3}$, it is easy to show that $Z_{d}$ in (5) is decreasing in $\left|c_{1}-c_{2}\right|$. So by (6), $Z_{d} \leq \frac{3}{2} c_{3}^{0}$ with equality if and only if $c_{1}=c_{2} \geq c_{3}$. Consequently this $d$ is E-optimal if and only if $N U_{1}=N U_{2}$ and $c_{1}-c_{3} \geq 0$.

\section{Discussion}

The E-optimality problem has been solved for three treatments and arbitrary numbers of levels $\left(b_{1}, \ldots, b_{n}\right)$ of crossed blocking factors. The surprising results are those for settings with one more experimental unit than needed for equal replication, for example, $5 \times 5$ or $7 \times 7$ row-column layouts. While in all cases the best strategy is to replicate as equally as possible, in these cases the assignment of the treatment with largest replication is made nonuniformly. In effect, E-efficiency increases as trace of the information matrix decreases to the extent allowed by (28) and (29).

In results to be reported elsewhere, we have solved the A-problem for three treatments in rowcolumn layouts. The two criteria sometimes agree, and sometimes disagree, on what design is best. The assignment conditions for A-optimality depend more crucially on the particular values of $b_{1}$ and $b_{2}$, and not just on the mod 3 value of their product. Common to the A-and E-problems is that 
maximal trace need not produce the best design. This phenomenon, now definitively established for $v=3$, is a chief reason that optimality theory for general $v$ is difficult for row-column designs. The statistical literature is sorely lacking in design results for settings where equal replication is not possible. This work is a step towards addressing that shortcoming, so that design theory can move closer to having a complete, flexible catalog of optimal designs available for experimenters. Though as seen here, optimality investigations without "nice" divisibility conditions on the design parameters can be both challenging and counterintuitive, progress can and should be made.

\section{ACKNOWLEDGEMENT}

J. P. Morgan was supported by National Science Foundation grant DMS01-04195.

\section{REFERENCES}

Bagchi, B. And Bagchi, S. (2001). Optimality of partial geometric designs. Annals of Statistics 29, 577-594.

Cheng, C.-S. (1978). Optimal designs for the elimination of multi-way heterogeneity. Annals of Statistics 6, 1262-1272.

Constantine, G. M. (1981). Some E-optimal block designs. Annals of Statistics 9, 886-892.

JACRoux, M. (1980). On the determination and construction of E-optimal block designs with unequal number of replicates. Biometrika 67, 661-667.

Morgan, J. P. (1997). On pairs of Youden designs. J. Statist. Plann. Inf. 60, 367-387.

Morgan, J. P. And Bailey, R. A. (2000). Optimal design with many blocking factors. Annals of Statistics 28, 553-577.

PARVu, V. (2004). Optimal blocking for three treatments and BIBD robustness: two problems in design optimality. Ph.D. Dissertation, Virginia Polytechnic Institute and State University, Blacksburg.

Sonnemann, E. (1985). U-optimum row-columns designs for the comparison of two treatments. Metrika 32, 57-63. 
Table 1: E-M-optimal assignments for 2 blocking factors

\begin{tabular}{|c|c|c|c|c|c|c|}
\hline$(2,2 ; 0,0)$ & $(2,5 ; 1,0)$ & $(2,8 ; 1,0)$ & $(2,11 ; 1,0)$ & $(2,14 ; 1,0)$ & $(2,17 ; 1,0)$ & $(2,20 ; 1,0)$ \\
$(4,4 ; 0,0)$ & $(4,7 ; 0,0)$ & $(4,10 ; 1,0)$ & $(4,13 ; 1,0)$ & $(4,16 ; 1,0)$ & $(4,19 ; 1,0)$ & $(5,5 ; 0,1)$ \\
$(5,8 ; 0,1)$ & $(5,11 ; 0,1)$ & $(5,14 ; 0,1)$ & $(5,17 ; 0,1)$ & $(5,20 ; 0,1)$ & $(7,7 ; 0,1)$ & $(7,10,1,0)$ \\
$(7,13 ; 0,1)$ & $(7,16 ; 2,0)$ & $(7,19 ; 0,1)$ & $(8,8 ; 0,1)$ & $(8,11 ; 2,0)$ & $(8,14 ; 1,1)$ & $(8,17 ; 1,1)$ \\
$(8,20 ; 1,1)$ & $(10,10 ; 0,1)$ & $(10,13 ; 0,1)$ & $(10,16 ; 2,0)$ & $(10,19 ; 1,1)$ & $(11,11 ; 0,2)$ & $(11,14 ; 0,2)$ \\
$(11,17 ; 0,2)$ & $(11,20 ; 2,1)$ & $(13,13 ; 0,2)$ & $(13,16 ; 1,1)$ & $(13,19 ; 1,1)$ & $(14,14 ; 0,2)$ & $(14,17 ; 2,1)$ \\
$(14,20 ; 2,1)$ & $(16,16 ; 0,2)$ & $(16,19 ; 0,2)$ & $(17,17 ; 0,3)$ & $(17,20 ; 0,3)$ & $(19,19 ; 0,3)$ & $(20,20 ; 0,3)$ \\
\hline
\end{tabular}

Table 2: E-M-optimal assignments for 3 blocking factors

\begin{tabular}{|c|c|c|c|c|c|c|}
\hline$(2,2,4 ; 0,1,0)$ & $(2,2,7 ; 1,1,0)$ & $(2,2,10 ; 1,1,0)$ & $(2,4,5 ; 1,0,1)$ & $(2,4,8 ; 0,1,1)$ & $(2,5,7 ; 0,1,1)$ & $(2,5,10 ; 0,1,1)$ \\
$(2,7,8 ; 1,0,2)$ & $(2,8,10 ; 0,2,1)$ & $(4,4,4 ; 0,1,1)$ & $(4,4,7 ; 1,1,1)$ & $(4,4,10 ; 0,1,2)$ & $(4,5,5 ; 0,1,2)$ & $(4,5,8 ; 1,0,3)$ \\
$(4,7,7 ; 0,2,2)$ & $(4,7,10 ; 0,2,3)$ & $(4,8,8 ; 1,2,3)$ & $(4,10,10 ; 0,3,3)$ & $(5,5,7 ; 1,2,2)$ & $(5,5,10 ; 0,2,3)$ & $(5,7,8 ; 2,2,3)$ \\
$(5,8,10 ; 2,3,3)$ & $(7,7,7 ; 2,2,2)$ & $(7,7,10 ; 2,2,3)$ & $(7,8,8 ; 2,3,3)$ & $(7,10,10 ; 2,3,3)$ & $(8,8,10 ; 3,3,3)$ & $(10,10,10 ; 3,3,3)$ \\
\hline
\end{tabular}

